\title{
Minimizing End-to-End Delay using Multipath Routing in Wireless Sensor Networks
}

\author{
S.Pratheema \\ Research Scholar \\ Department of CSE (PG) \\ National Engineering College, \\ K.R. Nagar, \\ Kovilpatti,Tuticorin District, \\ Tamilnadu, India
}

\author{
K.G.Srinivasagan \\ Professor and Head \\ Department of CSE (PG) \\ National Engineering College, \\ K.R. Nagar, \\ Kovilpatti,Tuticorin District, \\ Tamilnadu, India
}

\author{
J.Naskath \\ Research Scholar \\ Department of CSE (PG) \\ National Engineering College, \\ K.R. Nagar, \\ Kovilpatti,Tuticorin District, \\ Tamilnadu, India
}

\begin{abstract}
Some sensitive applications such as volcanic monitoring, fire detection data should be transmitted within a specified delay to the base station. Multipath-GT (Multipath - Generalized Topology) model uses an on-demand approach to estimate a delay based on processing time, packet loss rate between two neighbouring nodes. In existing work, if a node or link failure occurs multipath routing didn't spread traffic over alternate paths. This paper take a view that, when certain nodes and links become over-utilized and cause congestion, proposed work can spread traffic over alternate paths to balance the load over those paths and increase the degree of fault tolerance. The simulation results show that reduces the probability of communication disruption and data loss during link failures.
\end{abstract}

\section{Keywords}

Wireless Sensor Networks (WSN), Routing, Multipath, Alternate Path, Fault Tolerance.

\section{INTRODUCTION}

\subsection{Wireless sensor Network}

Wireless sensor networks (WSN) are now used in many applications including military, environmental, healthcare applications, home automation and traffic control. It consists of a large number of sensor nodes, densely deployed over an area. A wireless sensor network [1] typically consists of a very large number of small, inexpensive, disposable, robust, and lowpower sensor nodes working cooperatively. Wireless sensor network generally composed of a large number of distributed sensor nodes that organize themselves into a multi-hop wireless network. Each network is equipped with more than one sensors, processing units, controlling units, transmitting units etc.

Typically, the sensor nodes coordinate themselves to perform a common task. Sensor nodes are capable of collaborating with one another and measuring the condition of their surrounding environments. The sensed measurements are then transformed into [2] digital signals and processed to reveal some properties of the phenomena around sensors. Due to the fact that the sensor nodes in WSN have short radio transmission range, intermediate nodes act as relay nodes to transmit data towards the sink node using multipath. The deployment of sensor nodes based upon the application types.

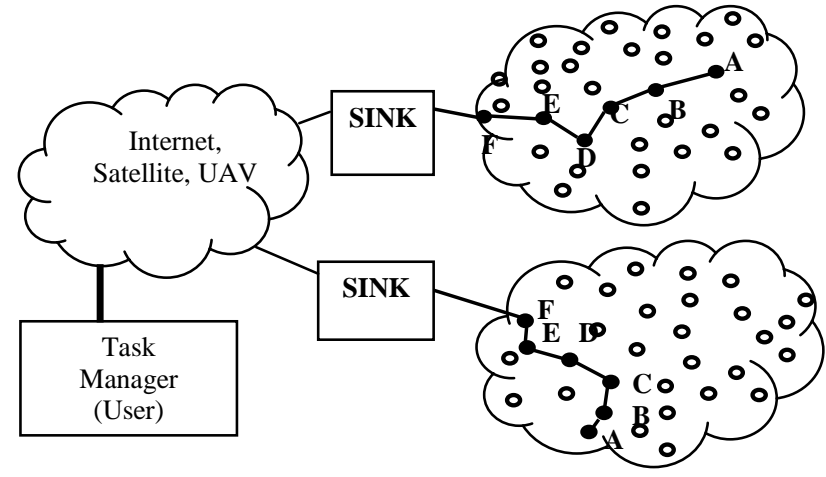

Figure 1: Basic structure of a Wireless sensor Network

In addition to basic routing, for mission-critical applications, Quality-of-Service (QoS) [5] routing protocols are needed to search for a path that can satisfy certain QoS requirements and constraints, such as bandwidth or data reliability.

\subsection{Routing Protocols for Sensor Network}

Routing in wireless sensor networks differs from conventional routing [3] in fixed networks in various ways: There is no infrastructure, wireless links are unreliable, sensor nodes may fail, and routing protocols have to meet strict energy saving requirements. Routing is a challenging task in WSNs because of their unique characteristics which makes it different from other wired and wireless sensor networks like cellular or mobile adhoc networks. Technically, sensor network nodes are limited [3] in respect to energy supply, computational capability and communication bandwidth. In order to prolong the lifetime of the sensor nodes, [4] designing efficient routing protocol is very critical. There are two important issues should be taken into account while designing a routing protocol for WSN.

- The level of power consumption at each stage of functionalities should be maintained.

- Tolerance of different types of failures should be achieved.

WSNs can be divided [6] into flat-based routing, hierarchicalbased routing, and location-based routing depending on the network structure. In flat-based routing, all nodes are typically assigned equal roles or functionality. A further classification 
criterion of flat based routing is the usage of messages: Routing method is called a single-path strategy, if there is only one instance of the message in the network at any time. Other forwarding strategies [10]can be classified as partial flooding and multi-path routing, depending on messages being forwarded to some neighbours in each routing step or when routing is performed along a few recognizable paths, respectively.

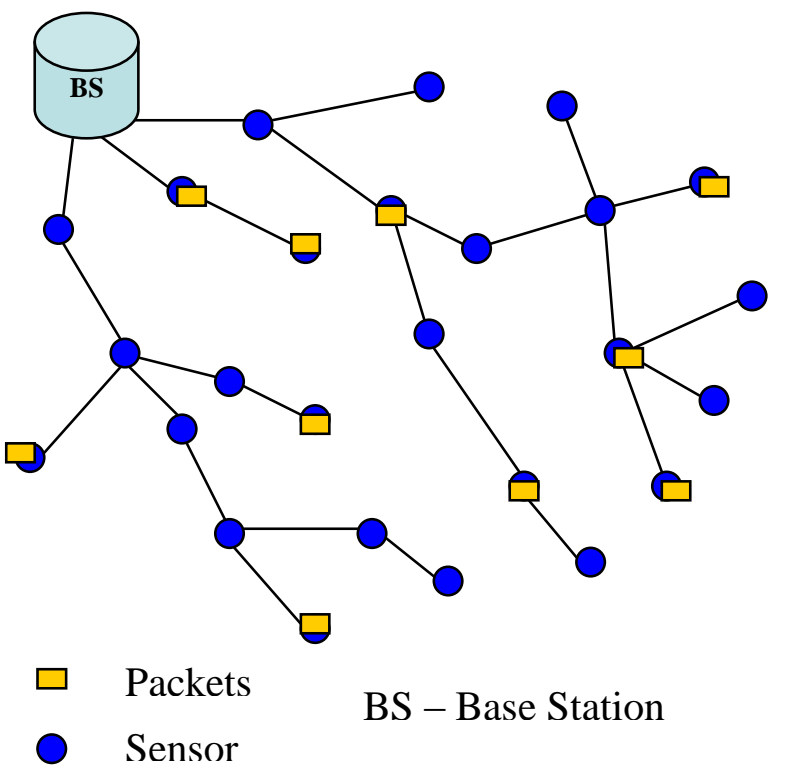

Figure 2 General Routing in Sensor Network

Multipath routing consists [11] of three components: route discovery, route maintenance and traffic allocation.

- Route discovery is the process of finding a route between two nodes.

- Route maintenance is the process finding broken routes, repairing them or finding a new route in the presence of a route failure.

- The main issues of traffic allocation strategy are to decide how the data will be sent and distributed in the network via available paths.

This paper proposes a work to design an alternate multipath routing protocol for WSNs when the nodes on the primary route fail. It helps to reduce the probability that communication is disrupted and data is lost in case of link failures. This scheme increases the degree of fault tolerance and it can be used for highly delay sensitive applications such as volcanic monitoring, fire detection in forest, where sensor nodes are deployed to monitor seismic activities, emission levels of volcanic craters etc.

The rest of the paper is organized as follows. Related works are given in Section II. In Section III describe overview of proposed scheme and multipath-GT routing protocol in detail. Performance comparisons and results are reported in Section IV. Finally, the conclusion of the proposed work is presented in Section V.

\section{RELATED WORK}

Routing in wireless sensor network is a challenging task due to various limitations such as bandwidth, power constraints, energy supply, etc. The routing protocols should make sure that the data packets are delivered to the base station fast and correct without or very little loss. Many existing routing protocols use multiple routing paths to forward packets. The benefits of using multipath include load balancing, resilience to unexpected node failures and increase the likelihood of reliable data delivery. Several single path and multipath routing protocols consider QoS routing guarantees. These protocols focus on a single service metric, such as reliability, delay or energy. Jongwon Choi [6] have proposed a lifetime enhancing and QoS provisioning routing protocol which distributes the traffic flow over multiple paths, by satisfying the different QoS requirements. It assumes that the network consists of two types of sensor nodes with two different QoS requirements. It focuses on satisfying QOS constraints while maximizing the lifetime of the networks. Yunfeng Chen [7] proposes a QOSMR: Quality Of Service Multipath Routing, a QoS incentive routing protocol for the purpose of reliability of packets delivery. It supports data transmission from more than one source to base station at the same time. The technique used by the proposed scheme is to create the clusters and represent the topology of the WSN involves the use of graph theory concepts.

Graph theory [7] can be used to create the sensor clusters and helps to identify the cluster head. This protocol uses the features of flat routing and hierarchical routing. In this scheme the routing is done by identifying the maximum energy possessing path links .This clustering scheme is to provide scalability. Kavitha.C [8] proposed a Hybrid Reliable Routing Technique (HRR) which focuses on two issues: organizing the network into clusters, changing the Cluster Head at the appropriate time and perform routing through the cluster heads to the sink consuming least energy. Gradient based algorithms such as Directed diffusion [9], GRAB [10] and GEAR [11] are data-centric in that all communication is for named data. All nodes in a directed diffusion-based network are application-aware. Data generated by sensor nodes is named by attribute-value pairs. A node requests data by sending interests for named data. Data matching the interest is then "drawn" down towards that node. Intermediate nodes might aggregate the data. An important feature of directed diffusion is that interest and data propagation and aggregation are determined by localized interactions.

Using multipath in wireless sensor networks can reduce frequent routing update and enhance data transmission rates. Additionally, it can provide an even distribution of traffic load over the network. This is of great benefit to balancing the energy consumption which is necessary for extending network lifetime. Most of multipath routing protocols are based on classic ondemand single path routing methods [12] [13] [14], such as AODV and DSR. They differ from each other on how to forward multiple route requests and how to select multiple routes. In some papers, node energy is also taken into consideration when constructing multiple paths [16] [17] [18]. The authors in [15] [18] proposed multipath routing schemes to distribute traffic among multiple paths instead of routing all the traffic along a single path. It intended to provide a reliable transmission environment with low energy consumption. The proposed protocol is capable to search multiple paths and aims to allocate the traffic rate to each path optimally. Both disjoint 
multipath and braided multipath algorithms are explored in [16]. Comparing disjoint multipaths to braided multipaths, braided multipaths have higher resilience to failures with fewer overheads. Network Quality Aware Routing [17] uses dynamic network quality factors such as link error rates and collision histories. It uses data centric on-demand method based on directed diffusion [9] to estimate minimum cost end to end routing path. It also extends network lifetime to prevent unnecessary energy consumption which will reduce packet losses and retransmission. Energy efficient multipath routing protocol [18] is capable of searching multiple paths and aims to allocate the traffic on each path optimally. It uses multiple paths between source and the sink which is intended to provide a reliable transmission environment with low energy consumption [16], by efficiently utilizing the energy availability of the nodes to identify multiple routes to the destination. Distributing the load to the nodes significantly impacts the system lifetime. It provides data synchronization and it has fault tolerance capability also achieves higher node energy efficiency.

A hierarchy-based multipath routing protocol called HMRP [19] which minimizes the path loading of the system by distributing the energy consumption among the nodes. HMRP employs hierarchical concept to construct whole sensor network. The wireless sensor network is initially constructed as a layered network. Based on the layered network, sensor nodes have multipath routes to the sink node through candidate parent nodes. HMRP supports multipath data forwarding, not use the fixed path. The energy consumption will be distributed and the lifetime of network will be prolonged. Finally, HMRP can support for multiple sink nodes situation.

\section{PROPOSED SYSTEM}

\subsection{Overview}

The proposed multipath-GT uses dynamic source routing algorithm, which provides soft QoS guarantees with respect to end-to-end reliability by discovering a set of multiple disjoint paths and transmitting data along these paths. In proposed work Graph theory can be used to find multipath to transfer data over the paths. Sensor network can be represented by a graph $G=(V$, $\mathrm{E})$, where the vertices $(\mathrm{V})$ represent the sensors and the set of links (E) represents the connections between vertices if the they are within the transmission range of each other. The flow diagram of proposed work is shown in Figure 3. The improvements of multipath-GT include the following steps multipath selection, delay metric analysis and backup route analysis. It also includes three points:

- $\quad$ First, the source node selects multipaths and that need to send data to base station.

- $\quad$ Second, source node chooses primary path, alternate path in advance among the selected multipaths and flow the data over primary paths.

- $\quad$ Third, if any node or link failure occurs, then alternate path is chosen to flow the data to avoid communication disruption and data lost.

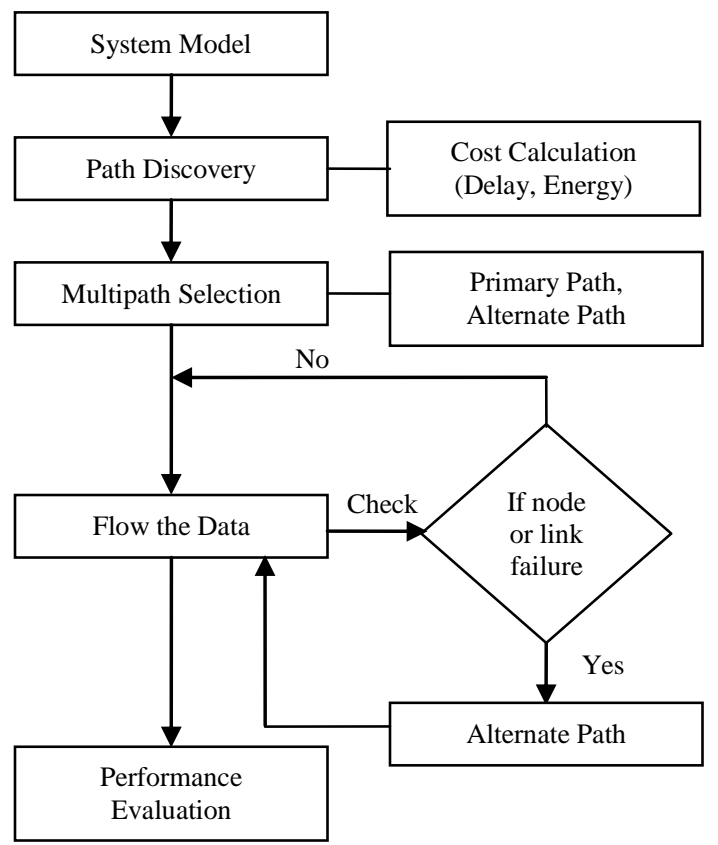

Figure 3 Flow of proposed Work

\subsection{Multipath-GT}

Multipath - GT (Multipath-Generalized Topology) model adaptively utilizes the network qualities including packet loss rates and delay histories in the route selection. It consists of two phases: route discovery phase and route maintenance phase. When an application uses multipath-GT for a route discovery, it supplies an end-to-end reliability requirement $p_{u}$, where $0 \leq p_{u} \leq 1$. Given this requirement, multipath-GT determines two parameters for the route discovery: (1) the number of paths it needs to discover; and (2) the lowest path reliability requirement that each search path must be able to provide in order to satisfy $p_{u}$. We refer to these two parameters as mo and $\Pi_{\text {lower }}$, respectively. These two parameters are decided based on the available state information. During route discovery phase neighbour discovery is performed when the sensor has no clue about the structure of its immediate surroundings. Figure 4 shows the scenario of neighbour discovery process of sensor nodes in the network. Every time a node wakes up, it broadcasts HELLO message. Only nodes that are also awake and within the communication range can hear this message and establish connections. The node out of communication range doesn't establish the connection with that node. After discovering the neighbours, routes are discovered using tree based search algorithm that calculates the path from one node to some pre-specified goal node. Figure 5 shows the scenario of route discovery process of sensor nodes in the network field. The tree based search algorithm finds shortest path using the qualities such as packet loss rate and delay. For that the following formula is used,

$$
f(n)=g(n)+h(n)
$$


Where $\mathrm{g}(\mathrm{n})$ is the packet loss rate from starting position to current location, $\mathrm{h}(\mathrm{n})$ is the estimated delay from current position to goal and $f(n)$ is the sum of $g(n)$ plus $h(n)$.

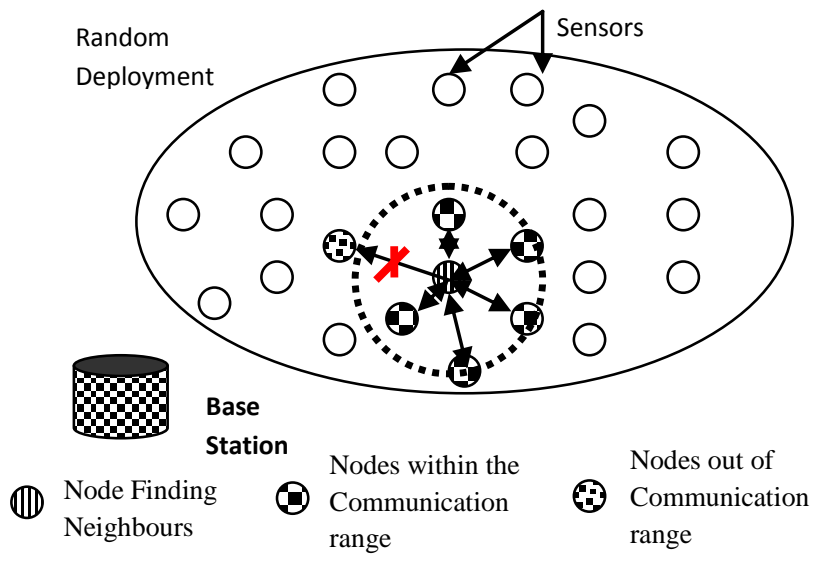

Figure 4 Neighbour Discovery

To initialize the route discovery process, route discovery algorithm specified in figure 6 was used. Algorithm performs some steps to construct various routes from source to destination. From these routes multiple paths and alternate paths are chosen to flow the data over those paths. After discovering all the possible paths, multipath identification is performed. In the proposed routing protocol the traffic is spread over the nodes lying on different possible paths between the source and the sink to provide a reliable transmission. To identify the multiple paths following path selection algorithm was used.

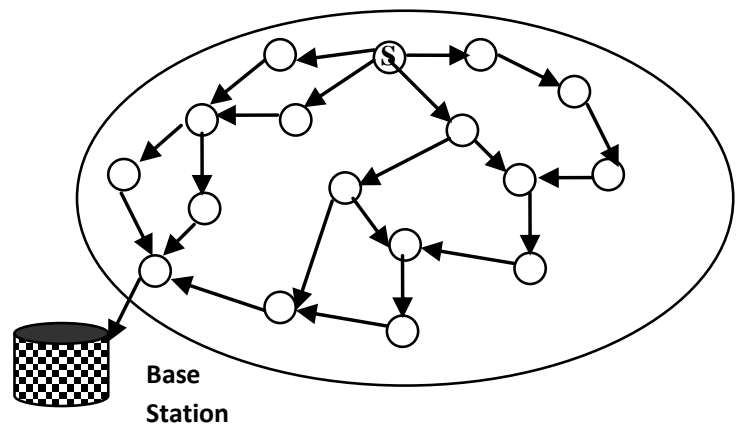

Figure 5 Route Discovery

The path selection algorithm is composed of two major steps. The first step is path-sorting algorithm, which is simply a sorting procedure that sorts all feasible paths (gathered from the RREQ messages) in a descending order according to their accumulated path reliabilities. We refer to the set of sorted paths as the candidate set. The second step is a disjoint path selection algorithm that selects a group of disjoint paths from the candidate set, such that this group of disjoint paths may collectively satisfy $\mathrm{Pu}$. We refer to this group of disjoint paths as the trace set. It takes the outputs of the path-sorting as its inputs, which include the candidate set and a set of path reliabilities corresponding to the candidate set (denoted as the reliability set).

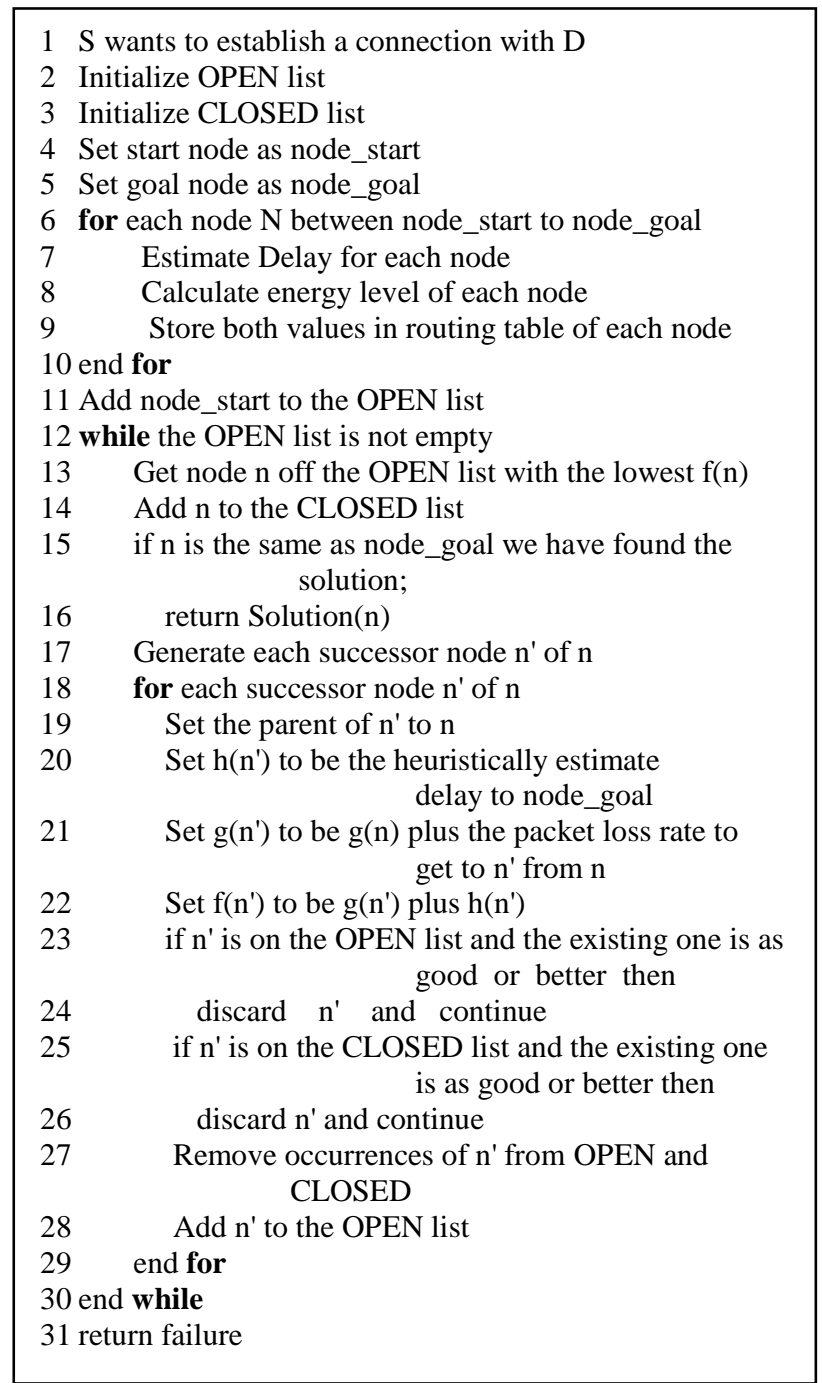

Figure 6 Algorithm for Route Discovery

The disjoint selection algorithm is generally a recursive algorithm. In each recursive step, the algorithm attempts to add a new path to the trace set, and the new path must be disjoint to all of the existing paths in the trace set. After the addition of the new path, the algorithm continues by calling itself to begin the next recursive step. As each recursive step begins, it obtains two pieces of information from the previous step:

- Which path in the candidate set that the current step should start to consider? This is denoted as the start index (si). This start index normally points to the path where all the preceding paths before the start index have already been considered by previous recursive steps.

- The end-to-end failure probabilities of the paths in the current trace set, denoted as fail Probability. The endto-end failure probability may be straightforwardly computed by multiplying path failure probabilities, each of which equals to one minus the corresponding path availability. 
If the current recursive step discovers that none of the paths in the candidate set starting from the start index is disjoint with those in the trace set, it then removes the paths that was previously added, and returns the control to the previous step (backtracking). In this case, the previous step continues by examining the paths after the one that has just been removed, and makes a recursive call when it finds another path that is disjoint to the trace set. The algorithm stops when an intermediate step discovers that the paths in the trace set have satisfied the requirement, which is equivalent to finding the endto-end failure probability less than the required reliability level (i.e. $1-p_{u}$ ). Otherwise, the algorithm returns a failure. Once the destination nodes have completed executing the path selection algorithm, it sends a set of RREP message through each disjoint path. Each RREP message stores its corresponding identifier pair, destination ID, as well as a disjoint path selected in the path selection algorithm. Each RREP messages traverses back to the source node using the path field of the message. Figure 7 shows the scenario of multiple paths selected by using path selection algorithm.

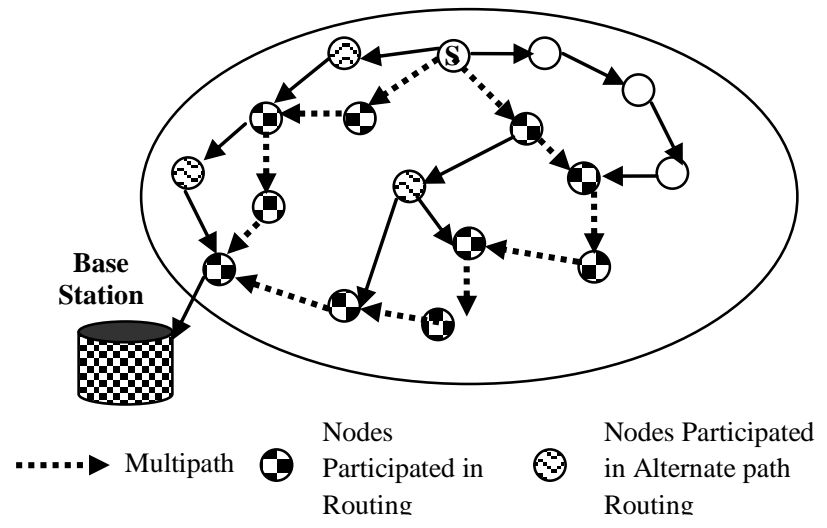

Figure 7 Multipath Selection

When the first RREP message arrives at the source node, the source node adds the path field into its route cache and immediately sends data using that path. When subsequent RREP messages arrives at the source node, the source node also adds their path fields into the route cache and may use these paths to send data packets. After each successful route discovery takes place, the source node can deliver its data to the destination node through a set of paths. However, these paths may break at any time instant due to the dynamic nature of network topology in wireless sensor networks. In the worst case, all paths may break and the source node can no longer transmit data to the destination node. In order to maintain a reliable and seamless network connection, route maintenance is necessary.

So that during maintenance phase when certain nodes and links become over-utilized and cause congestion, multi-path routing can spread traffic over alternate paths to balance the load over those paths. When end-to-end reliability is below the $\mathrm{Pu}$ requirement during the transmission, route maintenance needs to be performed. Rather than executing a route discovery again, Multipath-GT can select additional paths based on history information. Multi-path alternate routing can avoid congestion and improve performance. Multi-path alternate routing protocols can increase the degree of fault tolerance. Multipath-GT also uses tensor models for energy efficient multipath routing in large scale networks. Tensor models are used to increase the efficiency of network performance with the multipath routing. Now we focus on expected end to end delay. Multipath-GT uses a data centric approach to estimate an end-to-end delay based on processing time, propagation delay, packet loss rate, and the retransmission timeout between two neighbouring nodes.

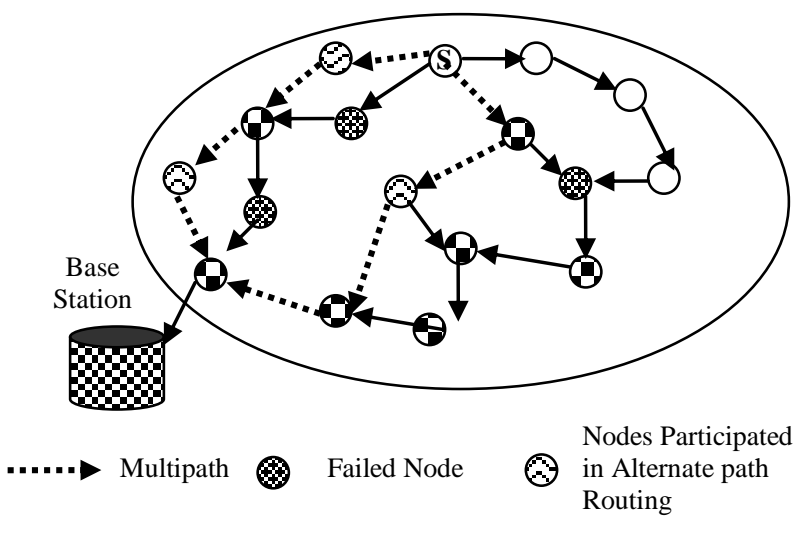

Figure 8 Backup route Selection

\section{PERFORMANCE EVALUATION AND SIMULATION RESULTS}

In this section, we compare the performance of standard DSR with multipath-GT algorithm through simulation experiments with different network scenes. The performance of multipathGT is evaluated by means of dynamic simulations with the help of the Network Simulator NS-2. We first describe the simulation setup and define the performance metrics. Then we show the simulation results.

\subsection{Simulation Setup}

Simulations have shown that Multipath-GT protocol can be considered as reliable. In our experiments, networks with a specified number of nodes are randomly distributed within an area of $1500 \times 300$. The IEEE 802.11 MAC protocol is used in the network. The number of nodes is varied as $50,60,70,80$, 90 and 100. Initially the nodes are placed randomly in the specified area. The following table summarizes the simulation parameters used.

Table 1: Simulation Parameters

\begin{tabular}{|c|c|}
\hline $\begin{array}{c}\text { Maximum Simulation } \\
\text { Time }\end{array}$ & $50 \mathrm{Sec}$ \\
\hline Area size & $1500 \times 300$ \\
\hline No of Nodes & $50,60,70,80,90,100$ \\
\hline Node Placement & Random Distribution \\
\hline Transmission Range & $250 \mathrm{~m}$ \\
\hline Traffic Type & CBR \\
\hline Initial energy & $100 \mathrm{~J}$ \\
\hline Transmit Power & $1.2 \mathrm{w}$ \\
\hline Receiving Power & $1.2 \mathrm{w}$ \\
\hline
\end{tabular}




\subsection{Performance Metrics}

The performance of Multipath-GT and standard DSR are compared by using the following metrics:

Packet Delivery Ratio: It is the ratio of the number .of packets received successfully and the total number of packets transmitted.

Average end-to-end delay: The end-to-end-delay is averaged over all surviving data packets from the sources to the destinations.

Throughput: It is the ratio of successfully received data packets by the base station to the total packets being sent from the source nodes.

Total Remained Energy: It is defined as the total unused energy level of nodes in the network.

\subsection{Simulation Results}

The simulation result shows that the performance comparison of Multipath-GT and DSR when the network size is varied. Figure 9 shows the throughput of two protocols under different network size. The throughput of multipath-GT is more compared to DSR based on varying number of nodes. The unit of throughput is Mbps. When the number of nodes is 80,90 and 100 the throughput of DSR protocol shows steadily increases, but multipath-GT shows rapid changes. The simulation results in figure 9 illustrates that when the number of nodes increases multipath-GT attained better throughput compared to standard DSR protocol.

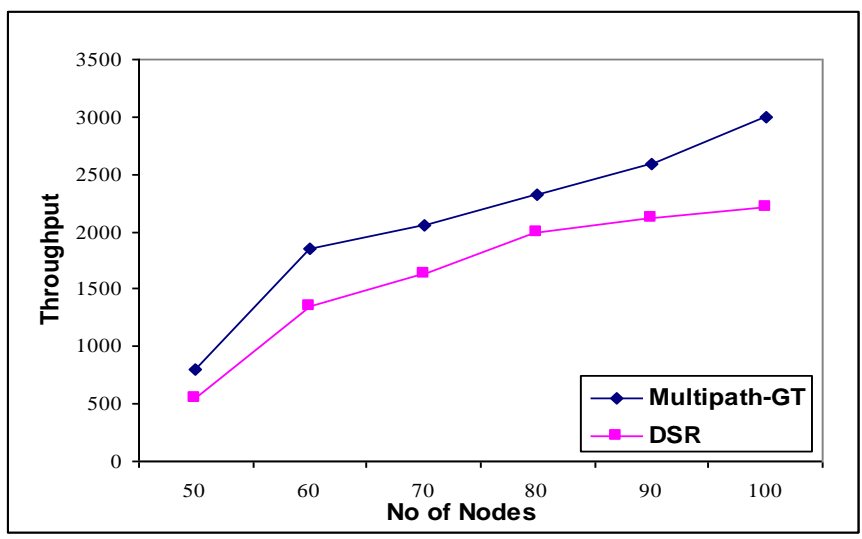

Figure 9 No of Nodes Vs Throughput

Then the end to end delay performance of two routing protocols is analyzed. The average packet delays under two schemes are plotted in Figure 10. The average end-to-end delay is the ratio of Sum of the delays of each packet received and number of packets received. Here, the delay of the packet refers to the difference between the time at which the packet reached the final destination minus the origination time of the packet. It shows that in DSR protocol when the number of nodes increases, delay also gradually increased. But in case of proposed multipath-GT routing protocol when the number of nodes increases, delay is not gradually increased rather than it decreases abruptly. So that compared to existing standard DSR protocol, multipath-GT reduces the average end to end delay up to $7 \%$. This result shows that multipath-GT has an ability to sustain application performance even for large node densities.

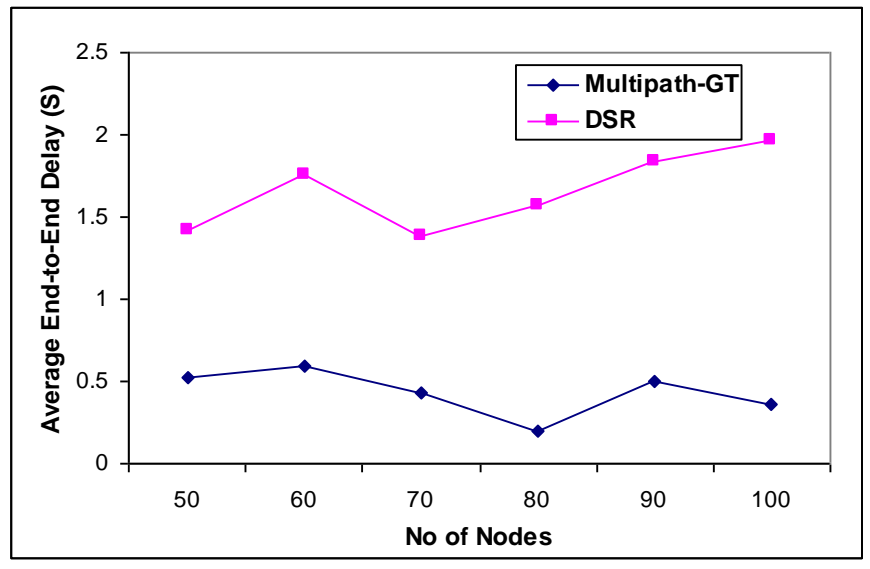

Figure 10 No of Nodes Vs Average End -to-End Delay

Figure 11 depicts packet delivery ratio of two routing protocols. It is found that the delivery ratio of multipath-GT routing protocol increases as the node density increases. When the node density is high there are more nodes available for data forwarding, and this increases the delivery ratio. DSR offers less packet delivery rates when network size increases. The proposed protocol has maintained constant delivery rates throughout the simulated scenarios because the multipaths are selected based on energy availability and minimum delay.

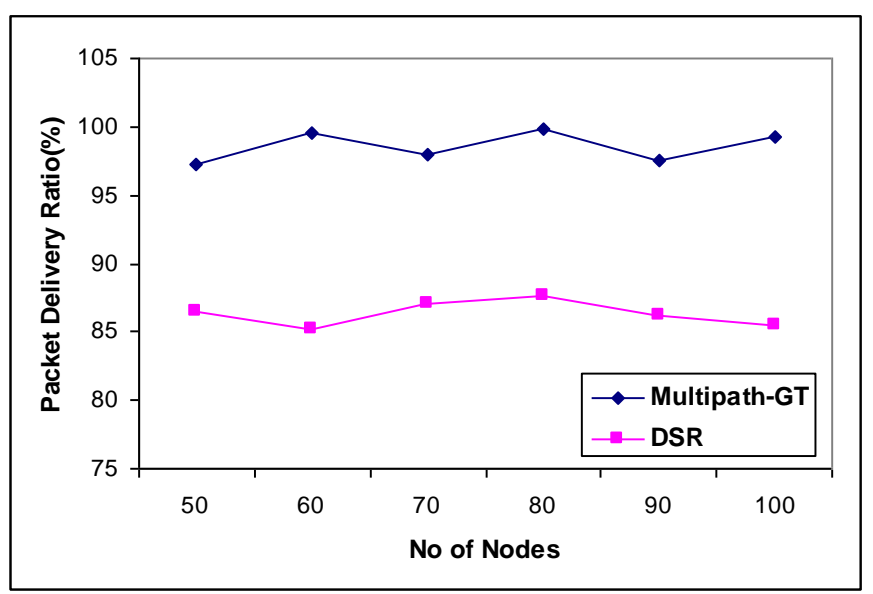

Figure 11 No of Nodes Vs Packet Delivery Ratio

Under energy constraints, it is crucial for sensor nodes to minimize energy consumption in communication. From the results shown in Figure 12, it is observed that there is a lower node energy consumption of multipath-GT routing over the other scheme. When compared to existing standard DSR protocol, the total remained energy of multipath-GT increases up to $15 \%$. Such experimental results demonstrate that the energy efficiency of multipath-GT is stable and it has the little impact by the increase of network size although the performance standard DSR degrades with larger network size. 


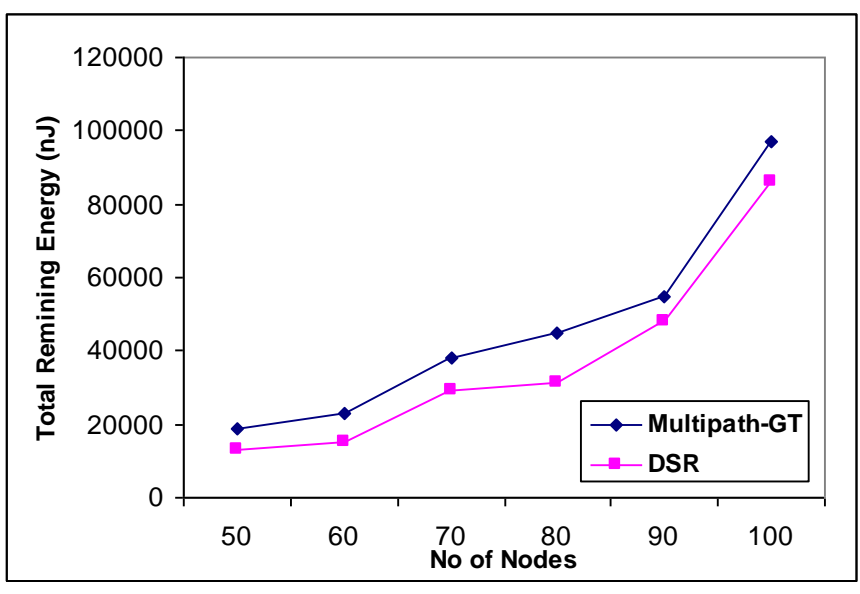

Figure 12 No of Nodes Vs Total Remining Energy

\section{CONCLUSION}

Routing is very important issue in wireless sensor networks. Many algorithms are proposed for efficient and reliable routing for delay sensitive applications. The main factors to be taken into consideration in case of WSN are battery life and fault tolerance. The multipath routing protocol is capable to search multiple paths and aims to allocate the traffic rate to each path optimally. Multipath-GT (Multipath - Generalized Topology) model adaptively utilizes the network qualities including packet loss rates, energy and delay histories in the route selection. The proposed multipath routing scheme distribute traffic among multiple paths instead of routing all the traffic along a single path and in case of link failure and retransmission alternate path is chosen to flow the data over the path. The simulated result shows that, the proposed scheme helps to reduce the probability that communication is disrupted and data is lost in case of link failures. The simulation result shows that when the network size varies multipath-GT achieves better packet delivery ratio and throughput. This scheme also increases the degree of fault tolerance.

\section{REFERENCES}

[1] Kazi Chandrima Rahman, "A Survey on Sensor Network", Journal of Computer and Information Technology(JCIT), ISSN 2078-5828

[2] Jamal N. Al-Karaki Ahmed E. Kamal, "Routing Techniques in Wireless Sensor Networks: A Survey", 2006

[3] Shio Kumar Singh, M P Singh, and D K Singh," Routing Protocols in Wireless Sensor Networks-A Survey", International Journal of Computer Science \& Engineering Survey (IJCSES) Vol.1, No.2, November 2010

[4] A. H. Azni, Madihah Mohd Saudi, Azreen Azman, and Ariff Syah Johari," Performance Analysis of Routing Protocol for WSN Using Data Centric Approach", World Academy of Science, Engineering and Technology 532009

[5] Roy Leung, Jilei Liu, Edmond Poon, Ah-Lot Charles Chan, Baochun Li," MP-DSR: A QoS-aware Multi-path Dynamic Source Routing Protocol for Wireless Ad-Hoc Networks"

[6] Jongwon Choi and Sehun Kim," A lifetime enhancing and QoS provisioning routing protocol for wireless sensor networks “,2008
[7] Yunfeng Chen and Nidal Nasser, "Enabling QoS Multipath Routing Protocol for Wireless Sensor Networks", IEEE Communications Society , ICC 2008

[8] Kavitha.C and Dr.K.V.Viswanatha," A Hybrid Reliable Routing Technique (HRR) for Wireless Sensor Network", IJCSNS International Journal of Computer Science and Network Security, VOL.9 No.3, March 2009

[9] C. Intanagonwiwat, R. Govindan, and D. Estrin, "Directed diffusion: a scalable and robust communication paradigm for sensor networks," in Proceedings of the 6th Annual International Conference on Mobile Computing and Networking (MOBICOM '00), pp. 56-67, Boston,Mass, USA, August 2000.

[10] B. Karp and H. T. Kung, "GPSR: Greedy perimeter stateless routing for wireless sensor networks," in the Proceedings of the 6th Annual ACM/IEEE International Conference on Mobile Computing and Networking (MobiCom '00), 2000.

[11] Y. Xu, J. Heidemann, and D. Estrin, "Geography-informed energy conservation for ad hoc routing," in the Proceedings of the 7th Annual ACM/IEEE International Conference on Mobile Computing and Networking (MobiCom'01), Rome, Italy, July 2001.

[12] M.K. Marina, and S.R. Das, "On-demand multipath distance vector routing in ad hoc networks," Proc. 9th IEEE Int. Conf. Network Protocols (ICNP), 2001, pp. 14-23.

[13] Amit N. Thakare, Mrs. M. Y. Joshi,’Performance Analysis of AODV \& DSR Routing Protocol in Mobile Ad hoc Networks", IJCA Special Issue on Mobile Ad-hoc Networks MANETs, 2010

[14] Sofiane Ouni, Jihen Bokri and Farouk Kamoun ," DSR based routing algorithm with delay guarantee for Ad Hoc networks“"JOURNAL OF NETWORKS, VOL. 4, NO. 5, JULY 2009

[15] R Vidhyapriya , Dr P T Vanathi ,"Energy Efficient Adaptive Multipath Routing for Wireless Sensor Networks", IAENG International Journal of Computer Science, 34:1, IJCS_34_1_8, publication: 15 August 2007

[16] Deepak Ganesan, Ramesh Govindan, Scott Shenker, Deborah Estrin, "Highly-Resilient, Energy-Efficient Multipath Routing in Wireless Sensor Networks", Mobile Computing and Communications Review, Volume 1, Number 2

[17] Jaewon Choi, Baek-Young Choi, Sejun Song, and KwangHui Lee," NQAR: Network Quality Aware Routing in Error-ProneWireless Sensor Networks", Hindawi Publishing Corporation EURASIP Journal on Wireless Communications and Networking, Volume 2010, Article ID 4097243

[18] A. V. Sutagundar, S. S. Manvi, Kirankumar. B. Balavalad, "Energy Efficient Multipath Routing Protocol for WMSN's", International Journal of Computer and Electrical Engineering, Vol. 2, No. 3, June, 2010

[19] Ying-Hong Wang, Chih-Hsiao Tsai and Hung-Jen Mao, "HMRP: Hierarchy-Based Multipath Routing Protocol for Wireless Sensor Networks", Tamkang Journal of Science and Engineering, Vol. 9, No 3, pp. 255_264 (2006) 